\title{
A preliminary study: The effect of ergonomic latex pillow on pain and disability in patients with cervical spondylosis
}

\author{
Fatemeh Fazli ${ }^{1}$, Behshid Farahmand*1, Fatemeh Azadinia ${ }^{1}$, Ali Amiri $^{2}$ \\ Received: 20 Jul 2017 \\ Published: 5 Sep 2018
}

\begin{abstract}
Background: Cervical pillows are considered to be part of the therapeutic strategy in cervical spondylosis, but there is little or no convincing scientific evidence on the effectiveness of these pillows in relieving pain and disability in these patients. Hence, this study investigated the effects of ergonomic latex pillow in conjunction with routine physiotherapy versus routine physiotherapy alone on disability and pain intensity in patients with cervical spondylosis.

Methods: 22 patients with cervical spondylosis in a single-blinded pilot study with a pre/post-test design were randomly assigned to experimental and control groups. Both groups received 12 sessions of physiotherapy for four weeks. In the experimental group, participants were given an ergonomic latex pillow to sleep it for four weeks. Participants in the control group slept on their own usual pillow. All the participants filled out the Neck Disability Index questionnaire, and scored their neck pain intensity on the Numerical Pain Rating Scale before and after the intervention. Changes of variables (pain and disability) within and between groups were compared by Paired T-test and the Independent Sample T-test, respectively via SPSS v.20 ( $<<0.05)$.

Results: Numerical Pain Rating Scale and Neck Disability Index scores were decreased statistically significant within groups. In the experimental group, neck disability scores were significantly lower than those in the control group ( $\mathrm{p}=0.038)$. However, pain intensity scores were not significantly different between groups.

Conclusion: Ergonomic latex pillows can significantly decrease disability related to neck pain in patients with cervical spondylosis after four weeks.
\end{abstract}

Keywords: Cervical spondylosis, Pillow, Neck pain, Disability

Copyright@ Iran University of Medical Sciences

Cite this article as: Fazli F, Farahmand B, Azadinia F, Amiri A. A preliminary study: The effect of ergonomic latex pillow on pain and disability in patients with cervical spondylosis. Med J Islam Repub Iran. 2018 (5 Sep);32:81. https://doi.org/10.14196/mjiri.32.81

\section{Introduction}

Cervical spondylosis, defined as a progressive intervertebral disc degeneration, is one of the most common disorders in the aging process (1), as various degrees of disc degeneration have been reported in $50 \%$ of people over 40 years of age, and $85 \%$ of people over 60 years of age (2). Spondylosis's symptoms may include axial neck pain, cervical radiculopathy, cervical myelopathy and sometimes a combination of these three syndromes (1).

Most practitioners and physical therapists recommend that patients use appropriate cervical pillows to relieve neck pain and stiffness (3) in addition to applying conservative therapeutic approaches such as exercise (4), electrotherapy modalities (5), cervical collars and traction (6).

Corresponding author: Behshid Farahmand, farahmand.b@iums.ac.ir

1. Department of Orthotics and Prosthetics, School of Rehabilitation Sciences, Iran University of Medical Sciences, Tehran, Iran.

2. Department of Physiotherapy, School of Rehabilitation Sciences, Iran University of Medical Sciences, Tehran, Iran.
It is thought that cervical pillows may allow disc rehydration through preserved cervical lordosis and by reducing the load exerted on the intervertebral discs. Therefore, it seems that cervical pillow and neck support can play an important role in the management of cervical disorders (7). In the current medical device market, cervical pillows come in so many different shapes and materials, all claiming to provide pain relief and improve the quality of sleep, which leave many patients confused as to which are most effective. Even though cervical pillows are widely used, most previous studies researching the effectiveness of cervical pillows have only been conducted in the laboratory, have solely tested healthy participants and/or have had defects in methodology (for example, short-term duration

$\uparrow$ What is “already known" in this topic:

Most practitioners and physical therapists recommend that patients use appropriate cervical pillows to relieve neck pain and stiffness only based on their own personal experiences and anecdotal evidence, rather than scientific evidence.

$\rightarrow$ What this article adds:

The ergonomic latex pillow is effective in reducing disabilities related to neck pain after four weeks of intervention in patients with cervical spondylosis. 
of exposure to the pillow or lack of a control group) (810). Given the particular weaknesses of these studies, there is very little scientific evidence in favor of the effectiveness of these pillows (11). Practitioners often recommend certain types of cervical pillows already on the market, mainly relying on their own personal experiences and anecdotal evidence (3). Widespread prescription of cervical pillows despite the lack of convincing scientific evidence regarding their efficacy in treating neck pain and neck disability index illustrates the need for additional and high-quality clinical trials.

A comparison of five different types of pillows in studies conducted by Gordon et al $(8,12,13)$ suggests that latex pillows are the most successful in improving the quality of sleep and reducing the waking symptoms in healthy subjects. However, the effectiveness of this type of pillow has not been studied in patients with cervical disorders. Hence, the present study aimed to investigate the effects of latex pillow in conjunction with standard physical therapy versus only standard physical therapy on pain, neck disability index and medication usage in patients with cervical spondylosis.

\section{Methods}

\section{Patients and study design}

This was a single-blinded pilot study with a pre and post-test design and a control group. This study was approved by Ethical Committee of Iran University of Medical sciences (9311502004), and was registered at the Iranian Registry of Clinical Trials with identification number IRCT2014021916643N1. Patients with cervical spondylosis were recruited from the outpatient physical therapy clinic of Hazrat-e-Rasul Akram Hospital. All subjects were screened by an experienced physical therapist and those who met inclusion criteria were selected for this study.

Patients met inclusion criteria if they were age 30 to 60 years of age; experienced chronic pain in the cervical region with or without pain radiation to their upper limbs; underwent a positive cervical compression and distraction test; had been diagnosed as having grade 2 or 3 of cervical spondylosis [based on existence of two out of four following radiographic changes in the cervical spine which include (1) reduction of intervertebral disc spaces, (2) sclerosis of subchondral bone, (3) reduced space between articular processes (facet joints), and (4) osteophyte formation between adjacent vertebral bodies, luschka or articular processes joints]; and obtain a score of $\geq 2$ on the Numerical Pain Rating Scale (NPRS), and a score of $\geq$ $20 \%$ on the Neck Disability Index (NDI) questionnaire. Patients with spinal canal stenosis, history of trauma or fracture and/or surgery of the cervical or cervicothoracic spine and history of using any kind of cervical pillow except a typical Iranian pillow (feather or usual cotton and sponge) were excluded. Moreover, patients were excluded if they received physical therapy or training because of neck pain during the last three months or prescribed to wear cervical collar. All participants signed the written informed consent form, and were randomly allocated to either an experimental group or control group.

\section{Interventions}

Both groups received equivalent standard physical therapy sessions twice weekly for four weeks. Each treatment session consisted of a five-minute ultrasound with $1 \mathrm{MHz}$ frequency and $1.5 \mathrm{~W} / \mathrm{cm}^{2} ; 20$ minutes of conventional transcutaneous electrical nerve stimulation (TENS) to the pain region (14); 20 minutes of superficial moist heat via hot pack (15); isometric and isotonic exercises of cervical muscles; and isotonic exercises of shoulder girdle muscles (16). All subjects were asked to follow their daily routine activities. Patients in the experimental group received an ergonomic latex pillow (WB001 LATEX PILLOW CE1674). The pillow's brand was removed and covered with a white pillowcase. The ergonomic latex pillow supported the subject in supine and side sleeping positions. Participants were given information and instructions on how to sleep on the pillow in the correct position. They were instructed to put only their head and neck on the pillow (not their shoulders with their chin in the hollow of the shoulder) (17). Patients in the control group were asked to continue sleeping on their usual pillow. None of subjects in both groups knew about the other group or group allocation.

\section{Measurement}

Demographic information and severity of disease were recorded. Primary outcome measures were pain intensity, functional disability, and medication usage. All the participants were asked to fill out the valid Neck Disability Index questionnaire (Iranian Version) and the Numerical Pain Rating Scale four weeks before the intervention, and a second time four weeks after the intervention. Neck Disability Index questionnaire assesses disability levels related to neck pain, and consists of 10 questions concerning sleeping, pain, concentration, personal care and activities of daily living. Participants were instructed to answer each question as it best pertained to their present situation. Each question had 0 to 5 scores and the maximum score of this questionnaire is 50, which would be reported as a percentage. Higher score indicates a higher level of disability. Numerical Pain Rating Scale had 11 points marked from " 0 " to " 10 ". Participants were asked to indicate the intensity of their highest, lowest and present levels of neck pain in the past 24 hours. In addition, the number of analgesic medications consumed was recorded during the 24 hours prior to the first and last sessions. The assessor was blinded to group allocation.

\section{Statistical analysis}

The data were analyzed by SPSS 16.00 version. To reduce the risk of bias, data were coded and the statistician was not aware of group allocation. The Shapiro-Wilk test was used for testing normality of variables. The independent sample T-test was applied for comparing demographic and baseline characteristics between groups. The Paired T-test was used to compare changes of outcome measures within each group, and the Independent Sample T-test was used to compare changes of outcome measures between the two groups. Non-parametric tests were applied just in case variables did not have a normal distribution. The 
Fisher's Exact test was used to compare analgesic numbers at baseline as well as its changes after four weeks between groups. P-value $<0.05$ was determined as the statistical significance in all analyses.

\section{Results}

Of 39 patients screened, 22 (1 male and 21females) were selected according to inclusion criteria. They were randomly allocated to two equal groups with a 1:1 ratio. Using the Shapiro-Wilk test, normal distribution was demonstrated in age, BMI, pain intensity and NDI scores variables, but not number of analgesics. Tables $1 \& 2$ presents baseline characteristics and clinical measurements of the subjects and also similarity between the groups $(p<0.05)$. Within groups, there were statistically significant decreases in both NDI $(p=0.001$ (experimental group), $\mathrm{p}=0.006$ (control group)) and NPRS scores $(\mathrm{p}=0.003$ (experimental group), $\mathrm{p}=0.001$ (control group)). Disability levels were significantly lower in the experimental group compared to the control group; however, the decrease in pain scores were not significantly different between two groups (Table 3). A significant decrease also was found in the number of analgesics consumed in the experimental group $(p=0.008)$, but the comparison between the groups was not statistically noticeable.

\section{Discussion}

Although the cervical pillow is considered a part of the therapeutic strategy for patients with cervical spondylosis (18), there is very little scientific evidence on the effectiveness of these pillows in relieving the symptoms of pain and disability in these patients. The aim of this study was to investigate the effect of ergonomic latex pillow combined with routine physical therapy versus routine physical therapy alone on neck disability, pain intensity and the medication usage in the patients with cervical spondylosis. The results of the present study demonstrated that pain intensity and disability in both groups were significantly lower after four weeks. Nevertheless, the levels of pain intensity, disability and the medication usage were lower in patients using the latex pillow compared to those using their regular pillow, though the treatment course in the experimental group was only significantly more effective in terms of the functional disability improvement.

Although it should be noted that any comparison between the findings of the present study and those of previous studies should be performed with caution due to differences in health status of participants and the types of pillows used in each study, it is apparent that the relative ineffectiveness of the ergonomic latex pillow in reducing pain immediately after completing the course of treatment in our study is in agreement with Bernateck et al. (19), who also found no difference in pain relief between the two groups after a course of intervention. However, further improvements in the symptoms were observed in long-term follow-up in the group receiving the pillow. A possible explanation for these findings is that the pillow needs time to produce noticeable changes in pain relief through restoration of intervertebral discs and muscles. In this context, the results of V. Desia et al. (20) revealed that the pain intensity in patients with cervical spondylosis was reduced after six weeks of using Bean Pillow. This finding can indicate the importance of time in the pillow's effectiveness. If the duration of our trial was longer than four weeks, we may likely have seen a significant increase in pain relief in our experimental group, though difference in the types of pillows used in our study and V. Desia's study should not be overlooked in terms of their influences on the outcomes of each study.

The neck pain relief scores reported by Erfanian et al. after four weeks usage of a semi-customized cervical pillow were contrary to the results of this study. At least some of the contradictory findings can be attributed to the difference in measuring the pain intensity in these two studies. In the study by Erfanian et al., the patients report-

Table 1. Severity of cervical spondylosis in groups

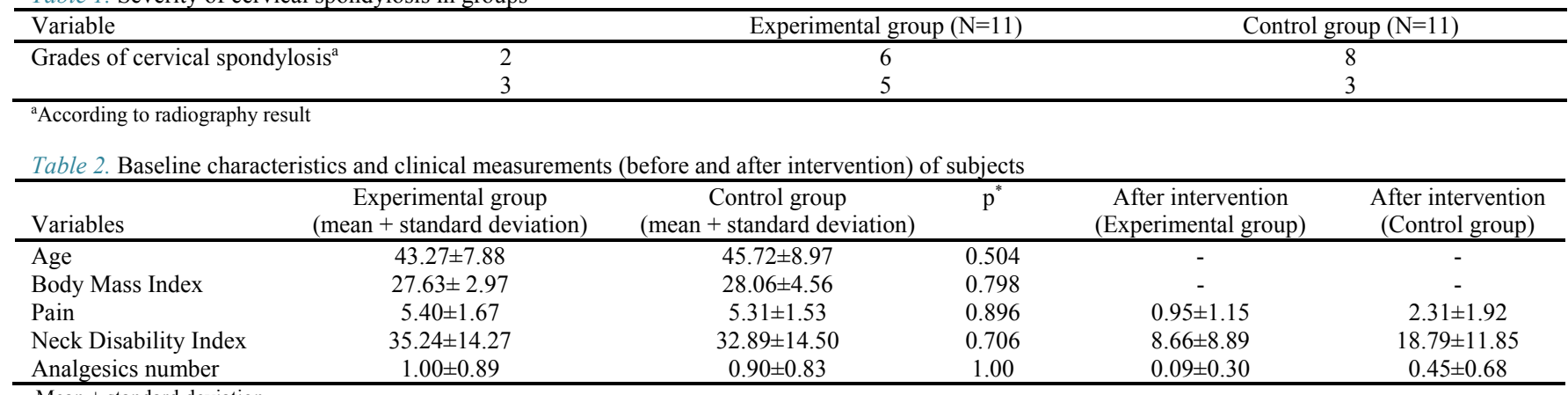

Mean \pm standard deviation

Table 3. Change of variables (before to after intervention) within and between groups

\begin{tabular}{lcccc}
\hline & $\begin{array}{c}\text { Experimental } \\
\text { variables }\end{array}$ & $\begin{array}{c}\text { Control } \\
\text { (mean + standard } \\
\text { deviation) }\end{array}$ & $\begin{array}{c}\mathrm{p}^{*} \\
\text { (Experimental group } \\
\text { after intervention) }\end{array}$ & $\begin{array}{c}\mathrm{p}^{*} \\
\text { (Control group after } \\
\text { intervention) }\end{array}$ \\
\hline Pain & $4.45 \pm 1.91$ & $3.00 \pm 2.16$ & $0.003^{*}$ & $0.001^{*}$ \\
Neck Disability Index & $26.58 \pm 15.05$ & $14.10 \pm 14.80$ & $0.001^{*}$ & 0.075 \\
Analgesics number & $0.90 \pm 0.70$ & $0.45 \pm 0.68$ & $0.008^{*}$ & $0.038^{*}$ \\
\hline
\end{tabular}

Analgesics number

$0.45 \pm 0.68$

0.008

0.311

Mean \pm standard deviation
$*$ The difference is significant at 0.05 level. 
ed their neck pain intensity at night before bedtime and in the morning after waking up; the morning pain levels had significantly decreased, but no significant change was observed in the pain intensity at night(17). In our study, patients reported the mean intensity of pain experienced in the past 24 hours. Given that the neck pain symptoms exacerbate after daily activities at the end of the day (21), this inclusion in the daily mean pain intensity might be the reason for non-significant changes in pain intensity among our patients, inconsistent with that of Erfanian et al (17).

It is noted that pain caused by degenerative disorders of the cervical spine arise from multiple anatomical sources including the facet joints, intervertebral discs, vertebral bodies, spinal ligaments and paraspinal musculature. Therefore, it makes the pain management challenging. In this point of view, one possible reason for non-significant difference between the groups is that the ergonomic latex pillow may decrease the pain through affecting the paraspinal musculature and supporting the spine regarding reduction of the abnormal loading and biomechanics, however there would be other sources that still generate pain.

In the present study, the superiority of the ergonomic latex pillow in reducing functional disability is in line with the findings of V. Desia et al. (20), Jagarnath (22) and Erfanian et al. (17). The significant reduction in functional disability may be attributed to improved quality of sleep in the participants, as it has been proven that quality of sleep could be directly correlated with physical health, mood and cognitive ability (23-25). Since previous studies have confirmed the positive effects of latex pillow on quality of sleep and waking symptoms $(8,12)$, it is likely that latex pillow would be effective in reducing functional disability by promoting the quality of sleep. The ergonomic latex pillow can maintain the head cooler so the subject feels a more pleasure sleep that causes a deeper sleep and may increase the quality of sleep which in turn can improve the subject's mood on the next day and improve the functional disability (26). In addition, this pillow with different heights (ergonomic) can support the head and neck in supine or side lying position. So it may be effective in improving the symptoms of the patients (27). On the other hand, our findings are contradictory to that of Lavin et al., who reported no significant decrease in disability index, in spite of improving the quality of sleep and reducing the waking symptoms. Aside from the difference in the types of cervical pillow between our two studies, a second possibility for the discrepancy is that our study design was different from that of Lavin et al. The present study was a pre and post-clinical trial with a control group. The experimental group employed the latex pillow during four consecutive weeks of intervention and the control group continued to use their usual pillow. However, in the study by Lavin, et al., each person used their usual pillow for one week, and then used a roll-pillow and water-based pillow for two weeks, without any washout period between pillow exposures; this design with short-term exposure duration to each pillow might also prevent its performance in improvement function in the aforementioned study (3). The authors acknowledge that the present study does have a notable limitation in term of its scope, being a preliminary investigation with only 11 participants in each group. Given that the amount of change in pain intensity in the experimental group was greater than that of the control group, it is likely that an increase in the number of participants could demonstrate a significant difference between two groups. Also we acknowledge that since most of participants were women, the findings cannot be generalized to men.

More studies are warranted to compare the ergonomic latex pillow regarding pain and neck disability index in a long term follow-up in both genders. Further research is warranted to identify efficacy of the latex pillow in terms of the objective and biomechanical outcome measures.

\section{Conclusion}

Ergonomic latex pillow can significantly decrease disability symptoms related to neck pain in patients with cervical spondylosis after four weeks of intervention. According to results of this study some symptoms of cervical spondylosis may decrease by using ergonomic latex pillow, thus it is recommended that these patients use an ergonomic latex pillow while receiving physical therapy to improve their neck disability symptoms.

\section{Acknowledgment}

We appreciate physical therapists in the physical therapy clinic of Hazrat-e-Rasul Akram Hospital of Iran University of Medical Sciences.

\section{Conflict of Interests}

The authors declare that they have no competing interests.

\section{References}

1. Ferrara LA. The biomechanics of cervical spondylosis. Adv Orthop. 2012;2012.

2. Kelly JC, Groarke PJ, Butler JS, Poynton AR, O'Byrne JM. The natural history and clinical syndromes of degenerative cervical spondylosis. Adv Orthop. 2012;2012.

3. Karim Y. The effect of various pillow types on cervico-thoracic and forward head posture in young adults [dissertation]. Durban University of Technology; 2014.

4. O'Riordan C, Clifford A, Van De Ven P, Nelson J. Chronic neck pain and exercise interventions: frequency, intensity, time, and type principle. Arch Phys Med Rehabil. 2014;95(4):770-83.

5. Kroeling P, Gross A, Graham N, Burnie SJ, Szeto G, Goldsmith CH, et al. Electrotherapy for neck pain. Cochrane Libr. 2013.

6. Hurwitz EL, Carragee EJ, van der Velde G, Carroll LJ, Nordin M, Guzman J, et al. Treatment of neck pain: noninvasive interventions. J Manip Physiol Ther. 2009;32(2):S141-S75.

7. Leilnahari K, Fatouraee N, Khodalotfi M, Sadeghein MA, Kashani YA. Spine alignment in men during lateral sleep position: experimental study and modeling. Biomed Eng Online. 2011;10(1):103.

8. Gordon SJ, Grimmer-Somers KA, Trott PH. Pillow use: the behavior of cervical stiffness, headache and scapular/arm pain. J Pain Res. 2010;3:137

9. Jeon MY, Jeong H, Lee S, Choi W, Park JH, Tak SJ, et al. Improving the Quality of Sleep with an Optimal Pillow: A Randomized, Comparative Study. Tohoku J Exp Med. 2014;233(3):183-8.

10. Gordon SJ, Grimmer-Somers KA, Trott PH. A randomized, comparative trial: does pillow type alter cervico-thoracic spinal posture when side lying? J Multidiscip Healthc. 2011;4:321.

11. Rooney M, Fess P, James D, Myers J, Murphy J, Taylor J, et al. Effect of mattresses and pillow designs on promoting sleep quality, 
spinal alignment and pain reduction in adults: Systematic reviews of controlled trials [dissertation]. Utica College; 2015.

12. Gordon SJ, Grimmer-Somers K, Trott P. Pillow use: the behaviour of cervical pain, sleep quality and pillow comfort in side sleepers. Man Ther. 2009;14(6):671-8.

13. Gordon SJ, Grimmer-Somers K. Your pillow may not guarantee a good night's sleep or symptom-free waking. Physiother Can. 2011;63(2):183-90

14. Bagheripour B, Kamyab M, Azadinia F, Amiri A, Akbari M. The efficacy of a home-mechanical traction unit for patients with mild to moderate cervical osteoarthrosis: A pilot study. Med J Islam Repub Iran. 2016;30:386-

15. Chung CT, Tsai SW, Chen CJ, Wu TC, Wang D, Lan $\mathrm{HCH}$, et al. Comparison of the intervertebral disc spaces between axial and anterior lean cervical traction. Eur Spine J. 2009;18(11):1669-76.

16. Akbari M, Bayat M. Effects of intermittent traction in patients with cervical osteoarthritis. Med J Islam Repub Iran. 2010;24(1):23-8

17. Soal LJ. Combining memory foam pillows with manipulation in the treatment of chronic cervical facet syndrome [dissertation]: Johannesburg Univ; 2014.

18. Caridi JM, Pumberger M, Hughes AP. Cervical radiculopathy: a review. HSS J. 2011;7(3):265.

19. Bernateck M, Karst M, Merkesdal S, Fischer MJ, Gutenbrunner C. Sustained effects of comprehensive inpatient rehabilitative treatment and sleeping neck support in patients with chronic cervicobrachialgia: a prospective and randomized clinical trial. Int $J$ Rehabil Res. 2008;31(4):342-6.

20. Desai KV, Rao SA. Effect of Bean pillow as an adjunct to exercises for neck pain in cervical spondylosis: A pilot study. Indian J Physiother Occup Ther. 2012;6(2):75.

21. Strom V, Roe C, Knardahl S. Work-induced pain, trapezius blood flux, and muscle activity in workers with chronic shoulder and neck pain. Pain. 2009;144(1-2):147-55.

22. Jagarnath K. The effectiveness of the Simmons Beautyrest ${ }^{\circledR}$ pillow in the management of chronic non-specific neck pain: a controlled clinical trial [dissertation]: Durban University of Technology; 2014.

23. Kyle SD, Morgan K, Espie CA. Insomnia and health-related quality of life. Sleep Med Rev. 2010;14(1):69-82.

24. Chen HL, Cai D. Body dimension measurements for pillow design for Taiwanese. Work. 2012;41 Suppl 1:1288-95.

25. Schutz TC, Andersen ML, Tufik S. The influence of orofacial pain on sleep pattern: a review of theory, animal models and future directions. Sleep Med. 2009;10(8):822-8. Epub 2009/02/07.

26. Liu SF, Lee YL, Liang JC. Shape design of an optimal comfortable pillow based on the analytic hierarchy process method. J Chiropr Med. 2011;10(4):229-39.

27. Her JG, Ko DH, Woo JH, Choi YE. Development and Comparative Evaluation of New Shapes of Pillows. J Phys Ther Sci. 2014;26(3):377. 